\title{
Lexicographic Strategic Games' Nonstandard Analysis
}

\author{
Guram N. Beltadze \\ Dept. of Control Systems, Georgian Technical University, Tbilisi, Georgia \\ E-mail: gbeltadze@yahoo.com
}

\begin{abstract}
A new concept of a mixed strategy is given for $m$-dimensional lexicographic noncooperative $\Gamma=\left(\Gamma^{0}, \Gamma^{1}, \ldots, \Gamma^{m-1}\right)$ game when on a set of pure strategies $m$-dimensional probability distributions are given. In this case each $\Gamma^{k} \quad(k=0,1, \ldots, m-1)$ criteria of $\Gamma$ game corresponds to its probability distributions on sets of pure strategies. Besides, a lexicographic $m$-dimensional order relation is given on set of $m$-dimensional probability distribution. The given construction is made by the methodology of nonstandard analysis Therefore, the given mixed strategy is called a nonstandard mixed strategy, and a lexicographic game in such strategies is called a nonstandard mixed extension. An equilibrium situation in mixed strategies is defined in $\Gamma$ game. A nonstandard mixed extension of lexicographic matrix games is studied thoroughly. In such games, necessary and sufficient conditions of the existence of a saddle point are proved. The analy zed examples show that if in a lexicographic matrix game doesn't exist a saddle point in standard mixed strategies then a saddle point maybe doesn't exist in nonstandard mixed strategies. If in a lexicographic matrix game doesn't exist a saddle point in standard mixed strategies then there can be existed a saddle point in nonstandard mixed strategies. Thus, lexicographic games' nonstandard mixed distribution is a generalization of a standard mixed extension.
\end{abstract}

Index Terms - Game, Noncooperative, Lexicographic, Strategy, Situation, Standard, Nonstandard, Equilibriu m, A Saddle Point

\section{Introduction}

Let discuss $m$-dimensional lexicographic noncooperative game [1-7] ,

$$
\begin{gathered}
\Gamma=<N,\left\{\chi_{i}\right\}_{i \in N},\left\{H_{i}\right\}_{i \in N}>\equiv \\
\left(\Gamma^{0}, \Gamma^{1}, \ldots, \Gamma^{m-1}\right)
\end{gathered}
$$

where every $i \in N=\{1, \ldots, n\}$ player's payoff vectorfunction $H_{i}$ we note by $H_{i}=\left(H_{i}^{0}, \ldots, H_{i}^{m-1}\right)$, its set of pure strategies we note by $\chi_{i}=\left\{x_{i}^{1}, \ldots, x_{i}^{p_{i}}\right\}$ and such kind of strategies set of situations - by $\chi=\prod_{i \in N} \chi_{i} \cdot i \in N$ is a player's standard mixed strategy

$$
X_{i}=\left(x_{i}^{1}, \ldots, x_{i}^{p_{i}}\right), \forall x_{i}^{k} \geq 0, x_{i}^{1}+\ldots+x_{i}^{p_{i}}=1,
$$
$i=1, \ldots, n$.

In (1) game the situation in standard mixed strategies has a form $X=\left(X_{1}, \ldots, X_{n}\right)$. Let note a set of situations in such strategies by $\mathrm{S}$.

On $\chi$ and S sets of situations the comparison of $H_{i}$ function's meanings takes place lexicographic weak $\geqslant{ }^{L}$ and strict preferences $-\succ^{L}$. For two $a=\left(a_{1}, \ldots, a_{m}\right) \quad$ and $\quad b=\left(b_{1}, \ldots, b_{m}\right) \quad$ vectors lexicographic preferance $a \succ^{L} b$ means that it fulfills one of the following $m$ conditions:

$$
\begin{aligned}
& \text { 1) } \left.a_{1}>b_{1} \text {; 2) } a_{1}=b_{1}, a_{2}>b_{2} ; . . ; m\right) \\
& a_{1}=b_{1}, \ldots, a_{m-1}=b_{m-1}, a_{m}>b_{m} \\
& \text { and } a \geqslant{ }^{L} b \text { if } a \succ^{L} b \text { or } a=b .
\end{aligned}
$$

Let note a set of equilibrium situations in $\Gamma$ games standard mexed extension by $\sigma(\Gamma)$. In such kind of games the main problem is that in every $\Gamma$ game there doesn't exist the equilib rium situation or mayby this set will be empty $-\sigma(\Gamma)=\varnothing$.

In the following article for $\Gamma$ game we take a player's new type of concept of a nonstandard mixed strategy, that is a $m$-dimensional probability distributions of $\Gamma$ game's on $\Gamma^{0}, \ldots, \Gamma^{m-1}$ criterion. By using such distributions payoffs' functions $H_{i}^{0}, \ldots, H_{i}^{m-1}$ reason, methodology of nonstandard analisis is capable [8]. Namely analisis of hyperreal infinitesimal nu mbers. 
For conducting such analysis it is necessary to define vectors lexicographic product operation by $\otimes$ notation.

$$
\begin{aligned}
& \text { Let we have } m \text {-dimensional vectors } \\
& a=\left(a^{0}, \ldots, a^{m-1}\right) \text { and } b=\left(b^{0}, \ldots, b^{m-1}\right) \text { we note } \\
& a \otimes b=\left(a^{0} b^{0}, a^{0} b^{1}+a^{1} b^{0},\right. \\
& a^{0} b^{2}+a^{1} b^{1}+a^{2} b^{0} \\
& \left.a^{0} b^{m-1}+\ldots+a^{m-1} b^{0}\right)
\end{aligned}
$$

$$
\left(a^{0} b^{0}, \sum_{k=0}^{1} a^{k} b^{1-k}, \sum_{k=0}^{2} a^{k} b^{2-k}, \quad, \sum_{k=0}^{m-1} a^{k} b^{m-1-k}\right)
$$

It is obvious, that $m$-dimensional $a \otimes b$ vector's components are the following form of one variable $m$. degree

$$
a(t)=a^{0}+a^{1} t+\ldots+a^{m} t^{m}
$$

and

$$
b(t)=b^{0}+b^{1} t+\ldots+b^{m} t^{m}
$$

coefficients of polynomials multiplication, only in difference with, that in the process of their multiplication $m$-degree polynomial can be conservated.

In the following lemma some essential properties of vectors lexicographic product is established.

Lemma 1.1 Lexicographic product's $\otimes$ operation defined by (2) has got the following properties:

1) Commutativity - $a \otimes b=b \otimes a$;

2) Associativity - $(a \otimes b) \otimes c=a \otimes(b \otimes c)$;

3) Distributivity of summation -

$$
(a+b) \otimes c=a \otimes c+b \otimes c
$$

4) "Monotonicity in two cases:

a) Weak monotonicity. If

$$
\begin{gathered}
\alpha=\left(\alpha^{0}, \ldots, \alpha^{m-1}\right) \in R_{+}^{m} \text { and }\left(a^{0}, \ldots, a^{m-1}\right){ }^{L} \preccurlyeq \\
\left(b^{0}, \ldots, b^{m-1}\right)\left(a^{L} \preccurlyeq b\right) \text {, then } a \otimes \alpha^{L} \preccurlyeq b \otimes \alpha ;
\end{gathered}
$$

b) Strict monotonicity.

$$
\begin{gathered}
\text { Let } \alpha=\left(\alpha^{0}, \ldots, \alpha^{m-1}\right) \in R_{+}^{m} \\
\left(a^{0}, \ldots, a^{m-1}\right) \succ^{L}(0, \ldots, 0) \text {. If }\left(a^{0}, \ldots, a^{m-1}\right){ }^{L} \prec \\
\left(b^{0}, \ldots, b^{m-1}\right) \text { and } a^{0} \neq 0 \text {, then } a \otimes \alpha^{L} \prec b \otimes \alpha
\end{gathered}
$$

Let note that in the conditions of b) case the requirement of difference from first components' xero is essential in $\left(a^{0}, \ldots, a^{m-1}\right)$ vector.

Let say that $(\Omega, A)$ is measurable space, where $\Omega \subset R^{m}$. Let discuss the transformation $\mu: A \rightarrow R_{m}^{+}$, that fulfills the following conditions: if $A_{i} \in A, \quad i=1,2, \ldots, \quad A_{i} \cap A_{j} \neq \varnothing, i \neq j$, $\bigcup_{i=1} A_{i} \in A \underset{\text {, then }}{\mu} \mu\left(\bigcup_{i=1} A_{i}\right)=\sum_{i=1} \mu\left(A_{i}\right)$

As a role of unit we take a vector $e=\left(1, \varepsilon, \varepsilon^{2}, \ldots, \varepsilon^{m-1}\right)$, where $\varepsilon$ is hyperreal infinitesimal number, and in addition the sum of $e$ components' is equal to number

$$
1+\varepsilon+\varepsilon^{2}+\ldots+\varepsilon^{m-1}=\frac{1-\varepsilon^{m}}{1-\varepsilon},
$$

and its standard part is equal to 1 .

In the second section of the article in $\Gamma=\left(\Gamma^{0}, \Gamma^{1}, \ldots, \Gamma^{m-1}\right) \quad$ game $\quad i \in N \quad$ player's nonstandard mixed strategy $\mathrm{X}_{i}$ is given by the form of a $p_{i} \times m$ matrix, where $p_{i}$ is an amount of $i \in N$ player's pure strategies. The set of $X_{i}$ strategies is noted by $\Sigma_{i}$. The set of situations in $\Gamma$ game is $\Sigma$, $\mathrm{X}=\left(\mathrm{X}_{1}, \ldots, \mathrm{X}_{n}\right) \in \Sigma$ is a situation in nonstandard mixed strategies, and $\Gamma_{\Sigma}$ is a mixed extension of $\Gamma$ game. The equilibrium situation is defined in $\Gamma_{\Sigma}$ game and their set is noted by $G\left(\Gamma_{\Sigma}\right)$.

In the third section a mixed extension $\Gamma_{\Sigma}$ of a matrix game and a saddle point in nonstandard mixed strategies are discussed. The property of transformation on nonstandard mixed strategies (lemma 3.1) and necessary and sufficient conditions of a saddle point in $\Gamma_{\Sigma}$ game are proved.

In the fourth section the examples of a lexicographic matrix games are discussed and it is shown that if in a game does not exist a saddle point in standard mixed strategies, then there can't be a saddle point in nonstandard mixed strategies either (example 4.1). If in a lexicographic matrix game does not exist a saddle point in standard mixed strategies, there can be a saddle point in nonstandard mixed strategies (example 4.2). 


\section{Lexicographic Noncooperative Nonstandard Mixed Extension}

Let define in lexicographic $\Gamma=\left(\Gamma^{0}, \Gamma^{1}, \ldots, \Gamma^{m-1}\right)$ game nonstandard mixed strategies and then make $\Gamma$ game's mixed extension. For this, in advance, note $m$. dimensional probability distribution on $\chi_{i}$ set in $\Gamma$ game for $i \in N$ player, that has $p_{i}$ amount of pure strategies in the following form:

$$
\begin{gathered}
X_{i}\left(x_{i}\right)=\left(X_{i}^{0}\left(x_{i}\right), \ldots, X_{i}^{m-1}\left(x_{i}\right)\right) \in R_{+}^{m}, \\
x_{i}=\left(x_{i}^{1}, \ldots, x_{i}^{p_{i}}\right),
\end{gathered}
$$

In this connection

$$
\sum_{l=1}^{p_{i}} X_{i}\left(x_{i}^{l}\right)=\left(1, \varepsilon, \varepsilon^{2}, \ldots, \varepsilon^{m-1}\right) \quad, i=1, \ldots, n,
$$

where $\mathcal{E}$ is a hyperreal infinitesimal number.

Let note $m$-dimensional probability distribution $X_{i}\left(x_{i}\right)$ on $\chi_{i}$ set of strategies for $i \in N$ player in a form of following matrix:

$$
\begin{aligned}
& X_{i}=\left(\begin{array}{c}
\left(X_{i}^{0}\left(x_{i}^{1}\right), \ldots, X_{i}^{m-1}\left(x_{i}^{1}\right)\right) \\
\ldots \ldots \ldots \ldots \ldots \ldots \ldots \ldots \\
\left(X_{i}^{0}\left(x_{i}^{p_{i}}\right), \ldots, X_{i}^{m-1}\left(x_{i}^{p_{i}}\right)\right)
\end{array}\right) \\
&=\left(X_{i}^{0}, \ldots, X_{i}^{m-1}\right), \\
& \sum_{l=1}^{p_{i}} X_{i}^{0}\left(x_{i}^{l}\right)=1 \sum_{l=1}^{p_{i}} X_{i}^{k}\left(x_{i}^{l}\right)=\varepsilon^{k}, k=1, \ldots, m-1 ., \\
& \text { This } \quad \mathrm{X}_{i} \quad \text { matrix is called } i \in N \quad \text { player's }
\end{aligned}
$$
nonstandard mixed strategy. It is obvious, that $\mathrm{X}_{i}$ strategy gives probability distributions on $\Gamma$ game's $\Gamma^{0}, \ldots, \Gamma^{m-1}$ criterion for $i \in N$ player.

Let note in $\Gamma$ game $i \in N$ player's nonstandard mixed set of strategies by ${ }^{\Sigma_{i}}$ and a set of situations by $\Sigma=\prod_{i \in N} \Sigma_{i}$ extension by

$$
\Gamma_{\Sigma}=<N,\left\{\Sigma_{i}\right\}_{i \in N},\left\{H_{i}\right\}_{i \in N}>
$$

Let in $\Gamma_{\Sigma}$ game $x=\left(x_{1}, \ldots, x_{n}\right) \in \chi$ is a situation in pure strategies, and $\mathrm{X}=\left(\mathrm{X}_{1}, \ldots, \mathrm{X}_{n}\right) \in \Sigma$ is a situation in nonstandard mixed strategies. Then in $\mathrm{X}$ situation $i \in N$ player's payoff is

$$
\begin{aligned}
& H_{i}(\mathrm{X})=\sum_{x \in \chi} H_{i}(x) \otimes \mathrm{X}(x) \\
& \sum_{x_{1} \in \chi_{1}} \ldots \sum_{x_{n} \in \chi_{n}} H_{i}\left(x_{1}, \ldots, x_{n}\right) \otimes \mathrm{X}_{1}\left(x_{1}\right) \otimes \ldots \mathrm{X}_{n}\left(x_{n}\right)
\end{aligned}
$$

For example let write (3) in case of two players i.e. when $N=\{1,2\}$. In the $\left(\mathrm{X}_{1}, \mathrm{X}_{2}\right)_{\text {situation for first }}$ player we have

$$
\begin{aligned}
H_{1}\left(\mathrm{X}_{1}, \mathrm{X}_{2}\right)= & \mathrm{X}_{1} \otimes H_{1} \otimes \mathrm{X}_{2}^{T}= \\
& \sum_{x_{1} \in \chi_{1}} \sum_{x_{2} \in \chi_{2}} \mathrm{X}_{1}\left(x_{1}\right) \otimes \mathrm{X}_{2}^{T}\left(x_{2}\right)
\end{aligned}
$$

According to the definition of multiplication of $\otimes$ operation and its 2) property for $x=\left(x_{1}, x_{2}\right) \in \chi$ we write:

$$
\begin{gathered}
\mathrm{X}_{1}\left(x_{1}\right) \otimes H_{1}(x)=\left(X_{1}^{0}\left(x_{1}\right) H_{1}^{0}(x), \ldots\right. \\
\left., X_{1}^{0}\left(x_{1}\right) H_{1}^{m-1}(x)+\ldots+X_{1}^{m-1}\left(x_{1}\right) H_{1}^{0}(x)\right), \\
\mathrm{X}_{1}\left(x_{1}\right) \otimes H_{1}(x) \otimes \mathrm{X}_{2}^{T}\left(x_{2}\right)= \\
\left(X_{1}^{0}\left(x_{1}\right) H_{1}^{0}(x) X_{2}^{0}\left(x_{2}\right), \ldots,\right. \\
X_{1}^{0}\left(x_{1}\right) H_{1}^{0}(x) X_{2}^{m-1}\left(x_{2}\right)+ \\
\left.X_{1}^{0}\left(x_{1}\right) H_{1}^{m-1}(x) X_{2}^{0}\left(x_{2}\right)\right) .
\end{gathered}
$$

For every $x_{1} \in \chi_{1}$ and $x_{2} \in \chi_{2}$ by summing given expressions we get:

$$
\begin{gathered}
\mathrm{X}_{1} \otimes H_{1} \otimes X_{2}^{T}=\left(X_{1}^{0} H_{1}^{0} X_{2}^{0}\right. \\
X_{1}^{0} H_{1}^{0} X_{2}^{1}+X_{1}^{1} H_{1}^{0} X_{2}^{0}+X_{1}^{0} H_{1}^{1} X_{2}^{0}, \ldots, \\
\left.X_{1}^{0} H_{1}^{0} X_{2}^{m-1}+\ldots+X_{1}^{0} H_{1}^{m-1} X_{2}^{0}\right)
\end{gathered}
$$

Analogically is for a second player too

$$
H_{2}\left(\mathrm{X}_{1}, \mathrm{X}_{2}\right)=\mathrm{X}_{1} \otimes H_{2} \otimes \mathrm{X}_{2}^{T} \text {. }
$$

Let note that $m$-vector's components are players payoffs certain sums in scalar $\Gamma^{0}, \Gamma^{1}, \ldots, \Gamma^{m-1}$ games. In these sums each summand has a form $X_{1}^{\alpha_{1}} H_{i}^{\alpha_{0}} X_{2}^{\alpha_{2}}$ $(i=1,2)$. Let change it by $H_{i}^{\alpha_{0}}\left(X_{1}^{\alpha_{1}}, X_{2}^{\alpha_{2}}\right)$ and say $\alpha_{0}+\alpha_{1}+\alpha_{2}=p$. After this it is obvious that $m_{\text {- }}$ 
vectors components are such summands $H_{i}^{\alpha_{0}}\left(X_{1}^{\alpha_{1}}, X_{2}^{\alpha_{2}}\right)$, for which $p$ numbers are constant and accordingly are equal to $p=0, p=1, \ldots, p=m-1$. Thus, in lexicographic bimatrix $\Gamma_{\Sigma}$ game $i \in\{1,2\}$ player's payoff in $\left(\mathrm{X}_{1}, \mathrm{X}_{2}\right)_{\text {situation is equal to }}$

$$
\begin{gathered}
H_{i}\left(\mathrm{X}_{1}, \mathrm{X}_{2}\right)=\left(H_{i}^{0}\left(X_{1}^{0}, X_{2}^{0}\right),\right. \\
\left.\sum_{p=1} H_{i}^{\alpha_{0}}\left(X_{1}^{\alpha_{1}}, X_{2}^{\alpha_{2}}\right), \ldots, \sum_{p=m-1} H_{i}^{\alpha_{0}}\left(X_{1}^{\alpha_{1}}, X_{2}^{\alpha_{2}}\right)\right) .
\end{gathered}
$$

If we note for any $n$ in $H_{i}^{\alpha_{0}}\left(X_{1}^{\alpha_{1}}, \ldots, X_{n}^{\alpha_{n}}\right)$ expression $p=\alpha_{0}+\alpha_{1}+\ldots+\alpha_{n}$, then analogically to (4) equality (3) has the following form:

$$
\begin{aligned}
H_{i}(\mathrm{X}) & =H_{i}\left(\mathrm{X}_{1}, \ldots, \mathrm{X}_{n}\right)= \\
& \left(H_{i}^{0}\left(X_{1}^{0}, \ldots, X_{n}^{0}\right),\right. \\
& \sum_{p=1} H_{i}^{\alpha_{0}}\left(X_{1}^{\alpha_{1}}, \ldots, X_{n}^{\alpha_{n}}\right), \ldots, \\
& \left.\sum_{p=m-1} H_{i}^{\alpha_{0}}\left(X_{1}^{\alpha_{1}}, \ldots, X_{n}^{\alpha_{n}}\right)\right), \quad i \in N .
\end{aligned}
$$

Let take a notation

$$
\mathrm{X} \|_{i} \stackrel{0}{\mathrm{X}}_{i}=\left(\mathrm{X}_{1}, \ldots, \mathrm{X}_{i-1}, \stackrel{0}{\mathrm{X}_{i}}, \mathrm{X}_{i+1}, \ldots, \mathrm{X}_{n}\right)
$$

then analogically to (4) we write:

$$
\begin{aligned}
H_{i}\left(\mathrm{X} \|_{i} \mathrm{X}_{i}\right) & = \\
& \left(H_{i}^{0}\left(X_{1}^{0}, \ldots, \mathrm{X}_{i}^{0}, \ldots, X_{n}^{0}\right),\right. \\
& \sum_{p=1} H_{i}^{\alpha_{0}}\left(X_{1}^{\alpha_{1}}, \ldots, \mathrm{X}_{i}^{\alpha_{i}}, \ldots, X_{n}^{\alpha_{n}}\right), \ldots, \\
& \left.\sum_{p=m-1} H_{i}^{\alpha_{0}}\left(X_{1}^{\alpha_{1}}, \ldots, \mathrm{X}_{i}^{\alpha_{i}}, \ldots, X_{n}^{\alpha_{n}}\right)\right)
\end{aligned}
$$

Definition 2.1 We say that a situation in nonstandard mixed strategies $\mathrm{X} \in \Sigma$ is an equilibrium situation in noncooperative $\Gamma_{\Sigma}$ game, if

$$
H_{i}(\stackrel{*}{\mathrm{X}}) \geqslant{ }^{L} H_{i}\left(\stackrel{*}{\mathrm{X}} \|_{i} \mathrm{X}_{i}\right), \forall i \in N, \forall \mathrm{X}_{i} \in \Sigma_{i}
$$

Let note by $G\left(\Gamma_{\Sigma}\right)$ a set of equilibrium situations in $\Gamma_{\Sigma}$ game.

With the preceding definitions and notations that are all about lexicographic noncooperative $\Gamma$ game's nonstandard mixed extension of $\Gamma_{\Sigma}$, it is clear to see the difficulty of the given apparatus. It seems that some classical results about scalar noncooperative games transfer to $\Gamma_{\Sigma}$ games, but some do not. Some important circumstances take place for $\Gamma_{\Sigma}$ games. We discuss some such kind of results for lexicographic matrix games [9].

\section{Lexicographic Matrix Game's Nonstandard Mixed Extension}

Let discuss lexicographic $p \times q$ matrix game $\Gamma=\left(\Gamma^{0}, \Gamma^{1}, \ldots, \Gamma^{m-1}\right)$ by matrix of payoff

$$
\begin{aligned}
H= & \left\{\left(a_{i j}^{0}, a_{i j}^{1}, \ldots, a_{i j}^{m-1}\right)\right\} \\
& i=1, \ldots, p ; j=1, \ldots, q
\end{aligned}
$$

In this game 1 and 2 players's commom (standard) mixed strategies are:

$$
\begin{aligned}
& X=\left(x_{1}, \ldots, x_{p}\right), \forall x_{i} \geq 0, x_{1}+\ldots+x_{p}=1 \\
& Y=\left(y_{1}, \ldots, y_{q}\right), \forall y_{j} \geq 0, y_{1}+\ldots+y_{q}=1
\end{aligned} ;
$$

In $\Gamma$ matrix game let note a set of equilibrium situation by $\sigma(\Gamma)$. As we have already stated above, this set could be empty $-\sigma(\Gamma)=\varnothing$.

Now discuss matrix $\Gamma=\left(\Gamma^{0}, \Gamma^{1}, \ldots, \Gamma^{m-1}\right)$ game in nonstandard mixed extension. Let note $i \equiv x_{i}=1, \ldots, p ; j \equiv y_{j}=1, \ldots, q$ and write $H$ vector in the following for

$$
\begin{aligned}
H=\left\{H\left(x_{i}, y_{j}\right)\right\}= & \left(\left\{H^{0}\left(x_{i}, y_{j}\right)\right\}, \ldots, .\right. \\
& \left.\left\{H^{m-1}\left(x_{i}, y_{j}\right)\right\}\right)
\end{aligned} .
$$

Analogically to a lexicographic noncooperative game, in the given matrix game players' nonstandard mixed strategies for 1 and 2 players accordingly have the following form: 


$$
\begin{aligned}
& X=\left(\begin{array}{c}
\left(X^{0}\left(x_{1}\right), \ldots, X^{m-1}\left(x_{1}\right)\right) \\
\ldots \ldots \ldots \ldots \ldots \ldots \ldots \ldots \\
\left(X^{0}\left(x_{p}\right), \ldots, X^{m-1}\left(x_{p}\right)\right)
\end{array}\right) \\
& =\left(X^{0}, \ldots, X^{m-1}\right) \text {, } \\
& \sum_{l=1}^{p} X^{0}\left(x_{i}\right)=1 \sum_{l=1}^{p} X^{k}\left(x_{i}\right)=\varepsilon^{k} \\
& k=1, \ldots, m-1 \text {. } \\
& \left.Y=\left(\begin{array}{c}
\left(\mathrm{Y}^{0}\left(y_{1}\right), \ldots, \mathrm{Y}^{m-1}\left(y_{1}\right)\right) \\
\ldots \ldots \ldots \ldots \ldots \ldots \ldots . . . \\
\left(\mathrm{Y}^{0}\left(y_{q}\right), \ldots, \mathrm{Y}^{m-1}\left(y_{q}\right)\right)
\end{array}\right)={ }^{0}, \ldots, Y^{m-1}\right) \\
& \sum_{j=1}^{q} Y^{0}\left(y_{j}\right)=1 \sum_{j=1}^{q} Y^{k}\left(y_{j}\right)=\varepsilon^{k} \\
& k=1, \ldots, m-1 \text {. }
\end{aligned}
$$

Let note $\Gamma$ matrix game's mixed extension in nonstandard mixed strategies as well as in the case of noncooperative games by $\Gamma_{\Sigma}$.

Let define in $\left(\mathrm{X}, y_{j}\right)$ situation the first player's payoff analogically to the operations held in (4) it is equal:

$$
\begin{aligned}
& H\left(\mathrm{X}, y_{j}\right)=\mathrm{X} \otimes H_{\cdot_{j}}= \\
& \quad\left(X^{0} H_{\cdot j}^{0}, \sum_{k=0}^{1} X^{1-k} H_{{ }_{j}}^{k}, \ldots, \sum_{k=0}^{m-1} X^{m-1-k} H_{{ }_{j}}^{k}\right) \\
& j=1, \ldots, q .
\end{aligned}
$$

We get the second player's payoff in the same way $\left(x_{i}, \mathrm{Y}\right)_{\text {situation }}$

$$
\begin{gathered}
H\left(x_{i}, \mathrm{Y}\right)=H_{i} \cdot \otimes \mathrm{Y}=\left(H_{i}^{0} \cdot Y^{0}, \sum_{k=0}^{1} H_{i}^{k} \cdot Y^{1-k}\right. \\
\left., \ldots, \sum_{k=0}^{m-1} H_{i}^{k} \cdot Y^{m-1-k}\right) \quad i=1, \ldots, p
\end{gathered}
$$

The first player's payoff in

$(\mathrm{X}, \mathrm{Y})$ situation analogically to (5) is equal

$$
\begin{aligned}
& H(\mathrm{X}, \mathrm{Y})=\mathrm{X} \otimes H \otimes \mathrm{Y}= \\
& \left(X^{0} H^{0} Y^{0}, \sum_{l=1} X^{\alpha_{1}} H^{\alpha_{2}} Y^{\alpha_{3}}, \ldots, \sum_{l=m-1} X^{\alpha_{1}} H^{\alpha_{2}} Y^{\alpha_{3}}\right)
\end{aligned}
$$

where $l=\alpha_{1}+\alpha_{2}+\alpha_{3}$.

From the (6) definition of noncooperative game's equilibrium situation we get the following definition.

Definition 3.1 In $\Gamma_{\Sigma}$ matrix game with matrix of $H$ payoff we call $\left({ }^{*},{ }^{*},\right)^{*}$ situation the equilibrium (sadle point), if for any $\mathrm{X}$ and $\mathrm{Y}$ nonstandard mixed strategies take place

$\Gamma \otimes H \otimes \mathrm{Y}^{*} \preccurlyeq{ }^{*} \mathrm{X} \otimes H \otimes \mathrm{Y}^{L} \preccurlyeq \stackrel{*}{ }{ }^{\mathrm{X}} \otimes H \otimes \mathrm{Y}$, or (7) accordingly

$$
\begin{aligned}
& \left(X^{0} H^{0} \mathrm{Y}^{* 0}, \ldots, \sum_{l=m-1} X^{\alpha_{1}} H^{\alpha_{2}} \mathrm{Y}^{* \alpha_{3}}\right)_{L} \\
& \left({ }^{*} H^{0} \mathrm{Y}^{* 0}, \ldots, \sum_{l=m-1} \mathrm{X}^{\alpha_{1}} H^{\alpha_{2}} \mathrm{Y}^{\alpha_{3}}\right)_{L} \\
& \left.{ }^{*}{ }^{\mathrm{X}^{0}} H^{0} \mathrm{Y}^{0}, \ldots, \sum_{l=m-1} \mathrm{X} H^{\alpha_{1}} \mathrm{Y}^{\alpha_{3}}\right)
\end{aligned}
$$

If the (8) conditions fulfill we write $(\mathrm{X}, \mathrm{Y}) \in G\left(\Gamma_{\Sigma}\right)$.

It is clear, that in noncooperative $\Gamma_{\Sigma}$ games the property of transformation on nonstandard mixed strategies is fulfilled. Let prove it for matrix $\Gamma_{\Sigma}$ game.

Lemma 3.2 Let in $\Gamma_{\Sigma}$ game are any nonstandard mixed $\mathrm{Y}=\left(Y^{0}, \ldots, Y^{m-1}\right)$ strategies of the second player's and $\left(a^{0}, \ldots, a^{m-1}\right)$ is a vector and inequalities are fulfilled

$$
H_{i} \otimes \mathrm{Y}^{L} \preccurlyeq\left(a^{0}, \ldots, a^{m-1}\right), i=1, \ldots, p .
$$

Then for any nonstandard mixed $\mathrm{X}=\left(X^{0}, \ldots, X^{m-1}\right)$ strategy of the first player the inequality is fulfilled

$$
\mathrm{X} \otimes H \otimes \mathrm{Y}^{L} \preccurlyeq\left(a^{0}, \ldots, a^{m-1}\right)
$$

Proof. According to (7) we write

$$
\begin{aligned}
& \left(H_{i}^{0} . Y^{0}, \sum_{k=0}^{1} H_{i}^{k} \cdot Y^{1-k}, \ldots,\right. \\
& \left.\sum_{k=0}^{m-1} H_{i}^{k} \cdot Y^{m-1-k}\right)_{L}\left(a^{0}, \ldots, a^{m-1}\right), i=1, \ldots, p .
\end{aligned}
$$

I.J. Intelligent Systems and Applications, 2013, 07, 1-8 
Each inequality for every $i=1, \ldots, p$ we multiply accordingly on $\mathrm{X}\left(x_{i}\right)=\left(X^{0}\left(x_{i}\right), \ldots, X^{m-1}\left(x_{i}\right)\right)$ According to lemma 1.1 inequalities are remained and by the definition of the operation ${ }^{\otimes}$ we get:

$$
\begin{aligned}
& \left(X^{0}\left(x_{i}\right) H_{i}^{0} \cdot Y^{0}, \sum_{l=1} X^{\alpha_{1}}\left(x_{i}\right) H^{\alpha_{2}} Y^{\alpha_{3}}, \ldots,\right. \\
& \left.\sum_{l=m-1} X^{\alpha_{1}}\left(x_{i}\right) H^{\alpha_{2}} Y^{\alpha_{3}}\right)_{L} \\
& \quad\left(a^{0} X^{0}\left(x_{i}\right), \sum_{k=0}^{1} a^{k} X^{1-k}\left(x_{i}\right), \ldots, \sum_{k=0}^{m-1} a^{k} X^{m-1-k}\left(x_{i}\right)\right) \\
& i=1, \ldots, p .
\end{aligned}
$$

Let sum these inequalities and take into account that in the components of vectors every summands except one that consists of $\varepsilon^{0}=1$ infinitesimal numbers. With the help of their ignorance we get:

$$
\begin{aligned}
& \left(X^{0} H^{0} Y^{0}, \sum_{l=1} X^{\alpha_{1}} H^{\alpha_{2}} Y^{\alpha_{3}}, \ldots, \sum_{l=m-1} X^{\alpha_{1}} H^{\alpha_{2}} Y^{\alpha_{3}}\right)_{L} \\
& \leqslant \\
& \left(a^{0}, \sum_{k=0}^{1} a^{k}\left(\sum_{i=1}^{p} X^{1-k}\left(x_{i}\right)\right), \ldots, \sum_{k=0}^{m-1} a^{k}\left(\sum_{i=1}^{p} X^{m-1-k}\left(x_{i}\right)\right)\right) \\
& =\left(a^{0} X^{0}\left(x_{i}\right), \sum_{k=0}^{1} a^{k} X^{1-k}\left(x_{i}\right), \ldots, \sum_{k=0}^{m-1} a^{k} X^{m-1-k}\left(x_{i}\right)\right) \\
& =\left(a^{0}, \sum_{k=0}^{1} a^{k} \varepsilon^{1-k}, \ldots, \sum_{k=0}^{m-1} a^{k} \varepsilon^{m-1-k}\left(x_{i}\right)\right) \\
& =\left(a^{0}, \ldots, a^{m-1}\right) .
\end{aligned}
$$

Hence (9) is proved.

Analogically will be the transformation on nonstandard mixed strategies in other eniqualities.

Theorem 3.1 For this purpose $(\mathrm{X}, \mathrm{Y}) \in G\left(\Gamma_{\Sigma}\right)$ it is necessary and sufficient for every $i=1, \ldots, p$ and $j=1, \ldots, q$ the following inequalities should be fulfilled

$$
H_{i} \otimes \stackrel{*}{\mathrm{Y}}{ }^{L} \leqslant \stackrel{*}{\mathrm{X} \otimes H \otimes} \otimes \stackrel{*}{\mathrm{Y}}^{L} \leqslant \stackrel{*}{\mathrm{X}} \otimes H \cdot_{j} .
$$

Proof. Sufficiency. If we use the both sides of (10) lemma 3.1, we get (3).
Necessity. In (8) conditions by following consequences let suppose that

$$
\begin{aligned}
& X=\left(\begin{array}{c}
\left(1, \varepsilon, \ldots, \varepsilon^{m-1}\right) \\
(0,0, \ldots, 0) \\
\ldots \\
(0,0, \ldots,))
\end{array}\right), \\
& X=\left(\begin{array}{c}
(0,0, \ldots, 0) \\
\left(1, \varepsilon, \ldots, \varepsilon^{m-1}\right) \\
\ldots \\
(0,0, \ldots,))
\end{array}\right), \ldots, \\
& X=\left(\begin{array}{c}
(0,0, \ldots, 0) \\
(0,0, \ldots, 0) \\
\ldots \\
\left(1, \varepsilon, \ldots, \varepsilon^{m-1}\right)
\end{array}\right) .
\end{aligned}
$$

Then, if we neglect summands, that take place $\varepsilon, \varepsilon^{2}, \ldots, \varepsilon^{m-1}$, the left side of (8) has a form

$$
\begin{aligned}
\left(H_{i}^{0} . Y^{0},\right. & \sum_{k=0}^{1} H_{i}^{k} \cdot Y^{1-k}, \ldots, \\
& \left.\sum_{k=0}^{m-1} H_{i}^{k} \cdot Y^{m-1-k}\right)_{L}{ }^{*} \mathrm{X} \otimes H \otimes \mathrm{Y}^{*}
\end{aligned}
$$

for any $i=1, \ldots, p$ and thus it fulfills (10) left part.

Analogically, if we put in (8) the second player's strategies by sequence

$$
\begin{aligned}
& \mathrm{Y}=\left(\left(1, \varepsilon, \ldots, \varepsilon^{m-1}\right)^{T},(0,0, \ldots, 0)^{T}, \ldots,(0,0, \ldots, 0)^{T}\right), \\
& \mathrm{Y}=\left((0,0, \ldots, 0)^{T},(1, \varepsilon, \ldots, \varepsilon)^{T}, \ldots,(0,0, \ldots, 0)^{T}\right) \\
& -\quad-\quad- \\
& \mathrm{Y}=\left((0,0, \ldots, 0)^{T},(0,0, \ldots, 0)^{T}, \ldots,(1, \varepsilon, \ldots, \varepsilon)^{T}\right)
\end{aligned}
$$

we get that it fulfills (10)'-s right part for every $j=1, \ldots, q$. The theorem is proved.

Corollary. Let $(\stackrel{*}{\mathrm{X}}, \stackrel{*}{\mathrm{Y}})$ is a saddle point in $\Gamma_{\Sigma}$ game $(\mathrm{X}, \mathrm{Y}) \in G\left(\Gamma_{\Sigma}\right)$. Then according to (10) that for every $i$ and $j$ 


$$
(\stackrel{*}{\mathrm{X}}, \stackrel{*}{\mathrm{Y}}) H_{i}^{0} \cdot \mathrm{Y}^{*} \leq \mathrm{X}^{0} H^{0} \mathrm{Y}^{0} \leq \mathrm{X}^{0} H^{0} \cdot{ }_{j}
$$

That means that $\left(\mathrm{X}^{0}, \mathrm{Y}^{0}\right)$ situation in standard mixed strategies is a saddle point in the first scalar matrix $\Gamma^{0}$ game - $\left(\mathrm{X}^{0}, \mathrm{Y}^{0}\right) \in \sigma\left(\Gamma^{0}\right)$ in lexicographic $\Gamma=\left(\Gamma^{0}, \Gamma^{1}, \ldots, \Gamma^{m-1}\right)$ matrix game.

\section{Examples}

On the basis of stated aparatus let conduct analysis of a lexicographic matrix $\Gamma=\left(\Gamma^{0}, \Gamma^{1}\right)$ game, which is given by matrix of payoff. Firstly, discuss a matrix game $\Gamma=\left(\Gamma^{0}, \Gamma^{1}\right)$, that was studied by P. Fishburn and showed that there is no saddle point in it $\sigma(\Gamma)=\varnothing_{[10]}$.

Example 4.1 A lexicographic matrix game $\Gamma=\left(\Gamma^{0}, \Gamma^{1}\right)$ is given by matrix of payoff

$$
H=\left(\begin{array}{ll}
(1,0) & (0,0) \\
(0,0) & (0,1)
\end{array}\right)
$$

Here $\sigma(\Gamma)=\varnothing$.

Let define for $\Gamma=\left(\Gamma^{0}, \Gamma^{1}\right)$ game nonstandard mixed strategies:

$$
\begin{aligned}
& \mathrm{X}=\left(\begin{array}{c}
(\alpha, \beta) \\
(1-\alpha, \varepsilon-\beta)
\end{array}\right), \\
& \mathrm{Y}=\left((\gamma, \delta)^{T},(1-\gamma, \varepsilon-\delta)^{T}\right)
\end{aligned}
$$

In this case the vectors $H_{i} \otimes \mathrm{Y}, \mathrm{X} \otimes H_{\cdot_{j}}$ and $\mathrm{X} \otimes H \otimes \mathrm{Y}$ have been already defined above as the following forms:

$$
\begin{aligned}
& H_{1} \otimes \mathrm{Y}=(\gamma, \delta), H_{2} \cdot \otimes \mathrm{Y}=(0,1-\gamma), \\
& \mathrm{X} \otimes H_{\cdot 1}=(\alpha, \beta), \mathrm{X} \otimes H_{\cdot_{2}}=(0,1-\alpha), \\
& \mathrm{X} \otimes H \otimes \mathrm{Y}_{=}(\alpha \gamma, \alpha \delta+\beta \gamma+\alpha \gamma+1-\alpha-\gamma) .
\end{aligned}
$$

According to the theorem 3.1 we write the following inequalities:

$$
\begin{aligned}
& \left.\begin{array}{c}
(\gamma, \delta) \\
(0,1-\gamma)
\end{array}\right\}{ }^{L} \leqslant(\alpha \gamma, \alpha \delta+\beta \gamma+\alpha \gamma+1-\alpha-\gamma) \\
& \qquad \begin{array}{c}
(\alpha, \beta) \\
(0,1-\alpha) .
\end{array}
\end{aligned}
$$

As in $\Gamma^{0}$ scalar game the first and the second players' optimal strategies are accordingly

$$
\mathrm{X}^{0}=\left(\alpha^{*}, 1-\alpha^{*}\right), \alpha^{*} \in[0,1]
$$

and $\mathrm{Y}^{*}=\left(\gamma^{*}, 1-\gamma^{*}\right), \gamma^{*}=0$, therefore according to (12) the following inequalities must by fulfilled

$$
\left.\begin{array}{c}
(0, \delta) \\
(0,1)
\end{array}\right\}^{L}{ }_{\leqslant}\left(0, \alpha^{*} \delta+1-\alpha^{*}\right)^{L} \leqslant\left\{\begin{array}{c}
\left(\alpha^{*}, \beta\right) \\
\left(0,1-\alpha^{*}\right),
\end{array}\right.
$$

but that is impossible. Thus, in the given game there also does not exist a saddle point in nonstandard mixed strategies.

Example 4.2 Let there is a lexicographic matrix game $\Gamma=\left(\Gamma^{0}, \Gamma^{1}\right)$ with a matrix of payoff

$$
H=\left(\begin{array}{ll}
(0,1) & (1,0) \\
(2,0) & (0,1)
\end{array}\right)
$$

There is no saddle point in it $-\sigma(\Gamma)=\varnothing$.

Let check up in the given game if there is a saddle point in it or not in nonstandard mixed strategies. For this let define (11) strategies and check up the theorem 3.1. As in $\Gamma^{0}$ game players' optimal strategies are

$$
\mathrm{X}^{*}=\left(\alpha^{*}, 1-\alpha^{*}\right)=\left(\frac{2}{3}, \frac{1}{3}\right)
$$

and

$$
\mathrm{Y}^{*}=\left(\gamma^{*}, 1-\gamma^{*}\right)=\left(\frac{1}{3}, \frac{2}{3}\right)
$$

therefore the condition (10) has the following form:

$$
\begin{aligned}
& \left.\begin{array}{l}
\left(\frac{2}{3}, \frac{1}{3}+\varepsilon^{*}-\delta^{*}\right) \\
\left(\frac{2}{3}, \frac{2}{3}+2 \delta^{*}\right)
\end{array}\right\}_{L}\left(\frac{2}{3}, \frac{4}{9}+\frac{2}{3} \varepsilon^{*}\right)_{L} \\
& \left\{\begin{array}{c}
\left(\frac{2}{3}, \frac{2}{3}+2 \varepsilon^{*}-2 \beta^{*}\right) \\
\left(\frac{2}{3}, \frac{1}{3}+\beta^{*}\right)
\end{array}\right.
\end{aligned}
$$


These inequalities fulfill when $\delta^{*}=0 ; \beta^{*}=\frac{1}{3}$, $\varepsilon^{*}=\frac{1}{3}$. Thus in $\Gamma=\left(\Gamma^{0}, \Gamma^{1}\right)$ game players' optimal nonstandard mixed strategies are accordingly to

$$
\stackrel{\mathrm{X}}{*}=\left(\begin{array}{c}
\left(\frac{2}{3}, \frac{1}{3}\right) \\
\left(\frac{1}{3}, 0\right.
\end{array}\right), \quad{ }^{*} \mathrm{Y}=\left(\left(\frac{1}{3}, 0\right)^{T},\left(\frac{2}{3}, \frac{1}{3}\right)^{T}\right)
$$

and $G\left(\Gamma_{\Sigma}\right) \neq \varnothing$. The first player's payoff is $H(\mathrm{X}, \stackrel{*}{\mathrm{Y}})=\left(\frac{2}{3}, \frac{7}{9}\right)$

Thus, offering construction considers necessities of vectorial criterion and is a generalization of a game's mixed extension. It is obvious that if $\sigma(\Gamma) \neq \varnothing$, then $G\left(\Gamma_{\Sigma}\right) \neq \varnothing$

\section{Conclusion}

By nonstandard mixed extension of lexicographic strategic games a generalization standard mixed extension has been conducted by using of nonstandard analysis, namely by analysis hyperreal infinitesimal numbers. It has been shown that if in the given game there exists an equlibrium situation in nonstandard mixed strategies, then in the same game there exists also an equilibrium situation in nonstandard mixed strategies. Such kind of analysis helps us to solve the problem about the existence of an equilib riu $m$ situation in games with the help of simple formal procedures

\section{References}

[1] G.N.Beltadze. Sets of equilibrium situations in lexicographic noncoalition games. Bullet in of the Academy of sciences of the Georgian SSR, 98, № 1, 1980, pp. 41-44 (in Russian).

[2] G.N.Beltadze. A mixed extension of finite noncoalition lexicographic games. Bullet in of the Academy of sciences of the Georg ian SSR, 98, № 2 1980, pp. 273-276 (in Russian).

[3] G.N.Beltadze. Analysis of the infinite dimensional lexicographic games. Bulletin of the Academy of sciences of Georg ian, 141, № 2 1991, pp. 241-244 (in Russian).

[4] G.N.Beltadze, A.L.Topchishvili. Multicriteria noncooperative games with strictly ordered criteria. A. Gopfert, J.Seelender, Chr. Tammer (Eds). Methods of Multicriteria Decision Theory, Proceedings of the 6 th Workshop of the DGOR Working Group Multicriteria Optimization and Decision, Frankfurt, 1997, pp. 69-86
[5] M. Salukvadze, G.N.Beltadze and F. Criado. Dyadic theoretical games models of decision making for the lexicographic vector payoffs. International Journal of information Technology and Decision Making, Vol. 8, Issue 2, 2009, pp. 193-216.

[6] G. N. Beltadze. Lexicographic non-cooperative game's mixed extension with criteria. International Journal of Systems and Software, ARPN Publishers, Vol 1, № 8, November 2011, pp. 247 250.

[7] G.N. Beltadze. Lexicographic Multistage Games with Perfect Information. Informational and Communication technologies-Theory and Practice: Proceedings of the International Scientific Conference ICTMC-2010 Devoted to the $80^{\text {th }}$ Anniversary of I.V. Prangishvili. Nova Publishers, 664 pp. USA, 2012. pp. 275-281.

[8] M. Davis. Applied Nonstandard Analisis. Courant Institute of Mathematical Sciences, New York University, 1977.

[9] G. N. Beltadze. On the reduction of the solution of a lexicographic matrix game to the solution of its square subgame. Bulletin of the Academy of sciences of the Georgian SSR, 104, № 1 (1981), pp 29-32 (in Russian)

[10] P. C. Fishburn. On the foundations of game theory: the case of non-Arximedean utilites. Inter. J.Game Theory, 1, № 2 (1972), pp. 65-71.

\section{Author's Profiles}

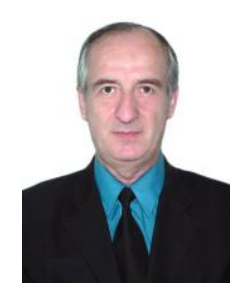

MR. Guram N. Beltadze: Full Professor at Informatics and Control Systems Faculty GTU. is a mathematician. Finished Tbilisi State University. He got a postgraduate education in the Academy of Sciences of the USSR in St.-Petersburg. His superrvisor was prof. N.N. Vorobiev. 1982 - Ph.D. at St.-Petersburg State University, 1992 - Dr. of Sci. of St.-Petersburg State University. Fields of research: Game Theory, Operations Research.

How to cite this paper: Guram N. Beltadze,"Lexicographic Strategic Games' Nonstandard Analy sis", International Journal of Intelligent Systems and Applications(IJISA), vol.5, no.7, pp.1-8, 2013. DOI: 10.5815/ijisa.2013.07.01 